\title{
Effects of Thermal Annealing on the Optical Properties of the Potassium Dihydrogen Phosphate Measured Using UV-Vis-NIR Spectroscopy
}

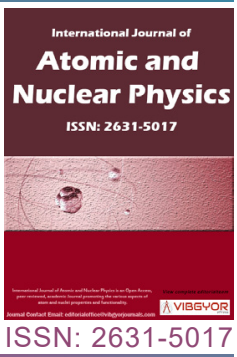

\section{Jianxiu Zhang, Zhongjun Tian\# and Kunpeng Wang*}

Zaozhuang University, Zaozhuang, China

"Equal Contribution

\begin{abstract}
Potassium Dihydrogen Phosphate (KDP) crystals have been successfully grown under different conditions such as slow growth rate $(2 \mathrm{~mm} /$ day $)$, rapid growth rate $(15 \mathrm{~mm} /$ day $)$, and deuterium (80\%) doping (DKDP). The extinction ratio, conductivity, absorption spectrum, laser damage threshold as well as optical scattering particles were fully investigated before and after thermal annealing in air, vacuum, $\mathrm{N}_{2}$ and $\mathrm{H}_{2}$, respectively. The correlations between optical properties and growth conditions were clarified. It was found that the laser damage threshold is proportional to the extinction ratio, optical transmittance, and however inversely proportional to the conductivity of the material. It was firstly convinced that the optical quality can be improved by thermal annealing in vacuum and $\mathrm{H}_{2}$, and however no obvious effect in air and $\mathrm{N}_{2}$.
\end{abstract}

Keywords

Potassium dihydrogen phosphate, Growth conditions, Optical properties, Thermal annealing

PACS

$81.10,42.70 . \mathrm{Hj}$

\section{Introduction}

Potassium Dihydrogen Phosphate (KDP) and deuterium doped KDP (DKDP) crystals have been widely used in optoelectronics, information, and national defense due to the advantages of the rapid growth rate, high laser damage threshold, large electro-optic and nonlinear optical coefficients $[1,2]$. Study on the growth and various properties for (D) KDP crystals has lasted more than 90 years [3]. There are two problems which are urgent to be solved and one is the rapid growth of large-aperture (D) KDP crystals (more than $500 \mathrm{~mm}$ along $a$ axis), and the other is about improvement of the optical and electrical qualities. Recently, 600 $\mathrm{mm}$-aperture KDP has been obtained during two weeks, and therefore the first problem was basically solved $[3,4]$. Unfortunately, the second problem, especially for the improvement of the optical homogeneity and laser damage threshold has not yet been well solved by now $[5,6]$. The optical and electrical properties of (D) KDP crystals could be greatly affected by the growth rate, solution conditions, deuterium concentration and so on $[7,8]$, and therefore study on the correlations between the growth conditions and optical properties may con-

*Corresponding author: Kunpeng Wang, Zaozhuang University, Zaozhuang, 277160, China

Accepted: October 06, 2020; Published: October 08, 2020

Copyright: (C) 2020 Zhang J, et al. This is an open-access article distributed under the terms of the Creative Commons Attribution License, which permits unrestricted use, distribution, and reproduction in any medium, provided the original author and source are credited.

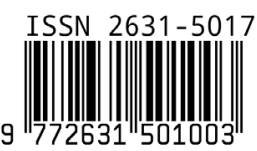

Zhang et al. Int J At Nucl Phys 2020, 5:022 
tribute to the improvement of the optical properties of (D) KDP. However, the changefulness of the growth conditions as well as numerous optical and electrical parameters of (D) KDP crystals made the correlation very complicated. In 2019, we designed and self-made a thermal annealing equipment, and simulated the temperature, stress distributions as well as airflow vectors in the equipment by the $\mathrm{Fi}$ nite Element Method [9]. This equipment has been used for annealing of all the (D) KDP samples on this study.

In this paper, the correlations between optical properties and growth conditions of (D) KDP were detailed clarified. Three growth conditions including traditional growth ( $1 \sim 5 \mathrm{~mm} /$ day), rapid growth ( $\geq 8 \mathrm{~mm} /$ day) and deuterium doped KDP(DKDP), as well as five optical parameters including extinction ratio, electrical conductively, transmittance spectra, laser damage threshold and distribution of the optical scattering particles, were taken into consideration[10]. In addition, the thermal annealing experiments in air, vacuum, $\mathrm{N}_{2}$ and $\mathrm{H}_{2}$ were also performed, and it was firstly found that the laser damage threshold is proportional to the extinction ratio, optical transmittance, and however inversely proportional to the conductivity of the material.

\section{Experimental Section Z Tian}

At first 20 samples were cut from KDP crystals grown from the three conditions with slow growth rate $(2 \mathrm{~mm} /$ day), rapid growth rate $(15 \mathrm{~mm} /$ day $)$, and deuterium doping (80\%), respectively. The (001) and (100) faces were polished, and a certain angle of each sample was chamfered in order to distinguish the $\pm a$ and $\pm c$ crystallographic directions. The samples were labeled as $X Y Z$, Where $X$ denotes the growth conditions such as slow growth rate $(S)$, rapid growth rate $(R)$ and deuterium doping (D), $Y$ denotes the annealing conditions such as in air (K), vacuum $(\mathrm{Z}), \mathrm{N}_{2}(\mathrm{~N})$, and $\mathrm{H}_{2}(\mathrm{H})$, Z denotes the serial-number or crystallographic directions. For example, $1 \sim 8$ means the measured order, and $a$ or $c$ refer to the crystallographic directions. The sizes and numbering of the 20 samples are shown as following: (a) 8 cuboid samples with sizes of 30 $\mathrm{mm} \times 40 \mathrm{~mm} \times 10 \mathrm{~mm}$ labeled as SK1, SK2, SZ3, SZ4, SN5, SN6, SH7 and SH8, along $a$ and $c$ directions respectively, were cut from KDP crystals with $2 \mathrm{~mm} /$ day growth rate. (b) 8 cuboid samples with sizes of $30 \mathrm{~mm} \times 40 \mathrm{~mm} \times 10 \mathrm{~mm}$ labeled as RK1, $\mathrm{RK} 2, \mathrm{RZ3}, \mathrm{RZ4}, \mathrm{RN} 5, \mathrm{RN} 6, \mathrm{RH} 7$ and RH8, along $a$ and c directions, respectively, were cut from KDP crystals with $15 \mathrm{~mm} /$ day growth rate. (c) 4 cuboid samples labeled as DK1, DK2, DH7 and DH8, with size of $20 \mathrm{~mm} \times 25 \mathrm{~mm} \times 10 \mathrm{~mm}$ were cut from DKDP crystals. Then another unpolished 20 samples with sizes of $10 \mathrm{~mm} \times 10 \mathrm{~mm} \times 3 \mathrm{~mm}$ were cut from KDP crystals grown under above three conditions for measurements of their conductivity. They were labeled according to the above-mentioned rules. Finally, thermal annealing experiments were performed on all the samples in the atmosphere of air, vacuum, $\mathrm{N}_{2}$ and $\mathrm{H}_{2}$, respectively, from $50{ }^{\circ} \mathrm{C}$ to $200^{\circ} \mathrm{C}$. Then the extinction ratio, conductivity, absorption spectrum, laser damage threshold as well as optical scattering particles were measured and compared before and after annealing.

In this paper, the extinction ratio was described as Ei/Eo. Eo is the energy received after the analyz$\mathrm{er}$, and $\mathrm{Ei}$ is the energy before the polarizer, which will be measured based on the reflected reference light. The extinction ratio is an important parameter for judging the quality of the KDP crystals. A large extinction ratio represents high optical quality. The conductivities were measured by using the conventional two-probe technique at room temperature. The absorption spectrum was measured by the UV-vis-NIR Spectroscopy. The laser damage threshold was measured by a Nd:YAG laser with a pulse width of $10 \mathrm{~ns}$, and a repetition of $1 \mathrm{~Hz}$ at the working wavelength $1064 \mathrm{~nm}$.

\section{Results and Discussions}

\section{Measurements of extinction ratio}

Five sites located at four corners and the central of each cuboid sample were selected to be measured. It was observed found that the measured extinction ratios are very different at different sites of each sample. The extinction ratios at central areas are generally higher than those at four corners, which indicate that the optical homogeneities in the center of the samples are better than that in edge areas. This is attribute to larger internal stresses which have been induced in the margin areas than in the center areas by the cutting and polishing processes. The comparisons of extinction ratios measured at different sites of each sample were shown in Figure 1a. In this figure $\mathrm{F}$ denotes the extinction ratio measured at the central, and $B, C, D$ as well as E denotes the four corners of the samples. From Figure 1 a, that the extinction ratios 


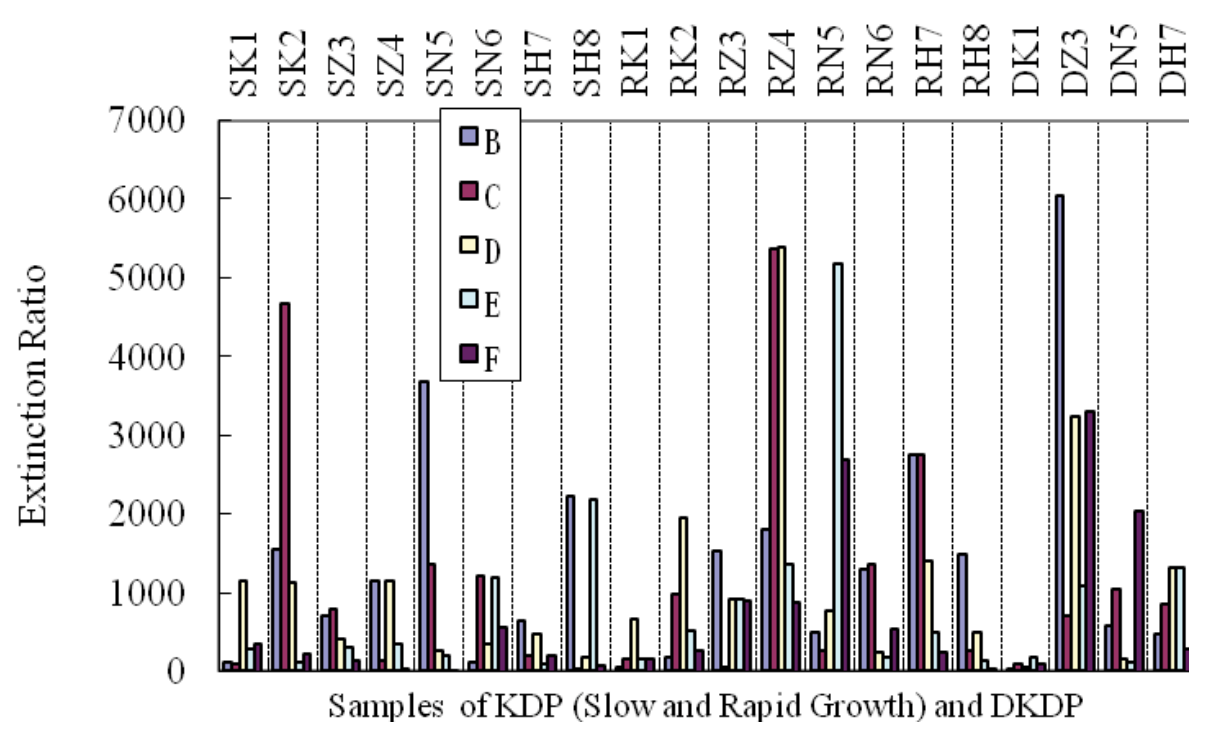

(a)

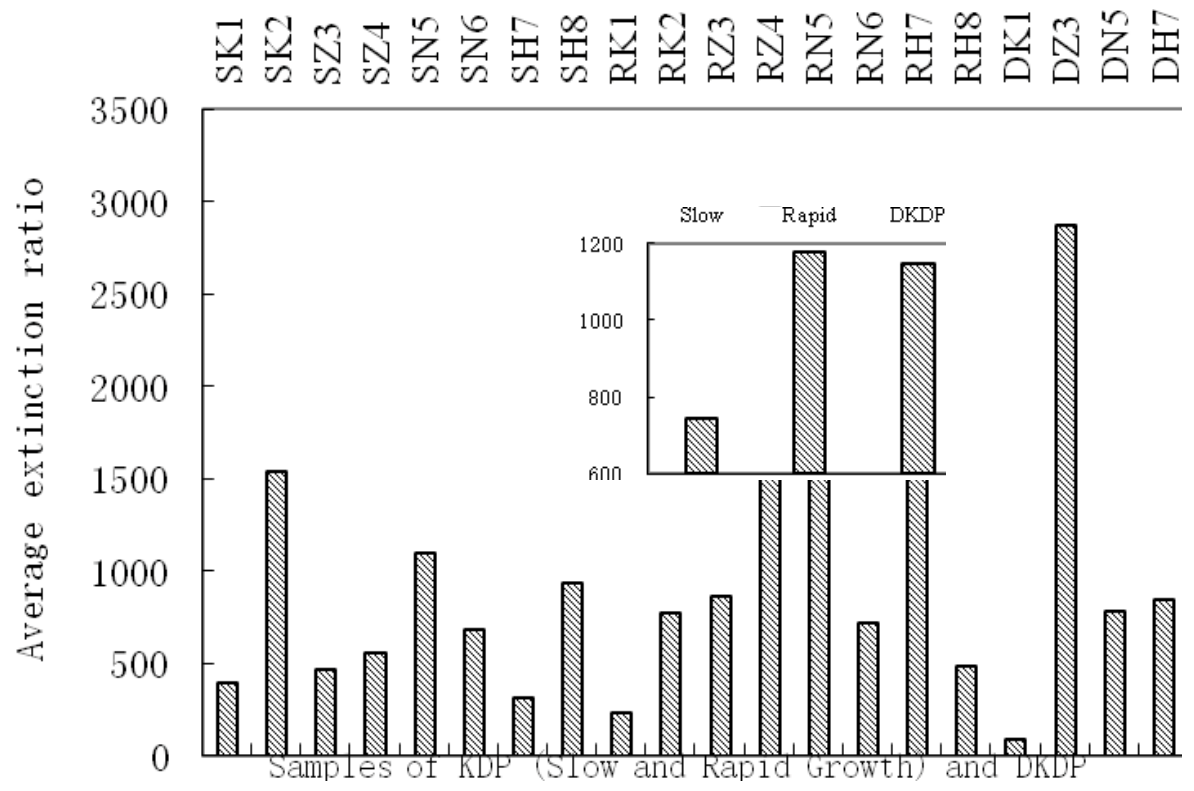

(b)

Figure 1: (a) The extinction ratio on different sites of slow or rapid grown KDP crystals; (b) The average extinction ratio on different 5 sites of each KDP crystal. The inset shows the extinction ratio of slow and rapid grown KDP and DKDP.

of crystals labeled as SK1, SK2 and SZ4 are lower than 350 at four corners, and higher than 1000 at the center of the samples. The trend of extinction ratios of the eight samples with rapid growth labeled as $\mathrm{R}^{* *}$ is upwards, and most of the measured values are larger than 1000 . The random distribution of the extinction ratios appears on the four DKDP crystals, and for instance five measured extinction ratios of the DK1 sample are very low, and whereas very high for DZ3. Neglecting the difference of extinction ratios located at different areas in one sample, the values out to compare the opti- cal homogeneities of crystals with different growth conditions was averaged and Figure $1 \mathrm{~b}$ shows the average extinction ratio on different 5 sites of each sample. It was seen From Figure $1 \mathrm{~b}$ that the optical homogeneities of crystals labeled as SK1, SK3, SK4, SN6, SH8, RK1, RH8 and DK1 are worse, that is to say, 5 traditional growth, 2 rapid growth and 1 DKDP crystals appear worse optical qualities and their extinction ratios are lower than 500 . Here it was noted that the optical qualities could be different due to sample processing although all the samples were cut from one crystal. So again, the 
average of 8 traditional, 8 rapid growth KDP and 4 DKDP crystals, and the results were shown in the inset of Figure $1 b$.

From the inset it can be learned that the average extinction ratio of crystals with traditional growth conditions is 746 , the rapid growth KDP is 1178 , and DKDP is 1146, which indicates that the optical homogeneities of rapid growth KDP and DKDP are better than traditional growth KDP. This result is obvious against common sense because it is generally believed that the optical quality would be perfect when the crystals growing with slow rate and high-purity. These measurement results also indicate that the effects of growth conditions on the qualities of DKDP crystals are very complicated, and one parameter is not enough to characterize the optical qualities of this crystal. So, the measurement results of other optical or electrical parameters of all samples will be given next.

\section{Conductivity}

The conductivities of all the samples were measured along $a$ and $c$ direction, respectively, using the conventional two-probe technique at room temperature. The results were shown in Figure 2. The conductivities along $a$ direction of five KDP crystals (62.5\% of all KDP samples) labeled as SK, $\mathrm{SZ}, \mathrm{SH}, \mathrm{RN}$ and $\mathrm{RH}$, and three DKDP crystals (75\% of all DKDP samples) labeled as DZ, DN and DH, are larger than those along $c$ directions. The conductivities of 4 traditional, 4 rapid growth KDP and 4
DKDP crystals were averaged and shown in Figure 2. It could be observed from this Figure that the conductivities were ranked in order of traditional growth KDP > rapid growth > DKDP. The conductivities along $a$ directions of rapid growth KDP and DKDP crystals were larger than those along $c$ directions, while for traditional growth KDP crystals, it was just the opposite. So, we drew a conclusion that the conductivities of DKDP crystals along $a$ direction are usually larger than those along $c$ directions since the electron transport channels are main along $a$ direction. In addition, it was noted that the extinction ratios of traditional growth KDP crystals are the lowest but their conductivities are whereas highest, and the extinction ratios of DKDP are larger but the conductivities are whereas lower. The results indicated that the extinction ratio is approximately inversely proportional to the conductivity of the samples.

\section{Optical transmittance}

Transmittance spectra of all samples were measured along $a$ and $c$ directions, respectively and shown in Figure 3. It can be seen that the whole transmittance range can be divided into four spectral regions, such as $200 \sim 400 \mathrm{~nm}(\mathrm{I}), 400 \sim 850 \mathrm{~nm}$ (II), $850 \sim 1200 \mathrm{~nm}$ (III) and $1200 \sim 1800 \mathrm{~nm}$ (IV).

For region I, the transmittances of DKDP and traditional growth KDP were higher than those of rapid growth KDP, and they were ranked in order of traditional growth KDP (measuring along $c$ di-

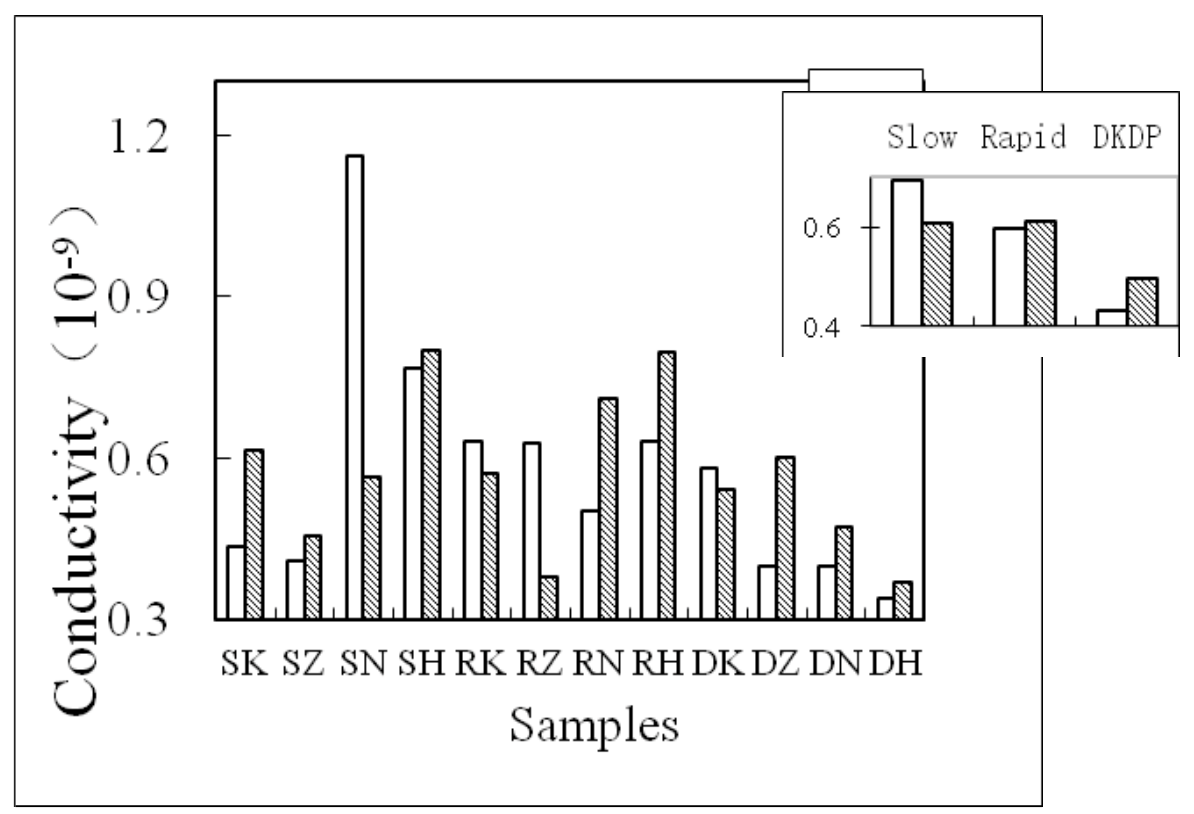

Figure 2: Conductivity of traditional, rapid growth KDP and DKDP crystals. 


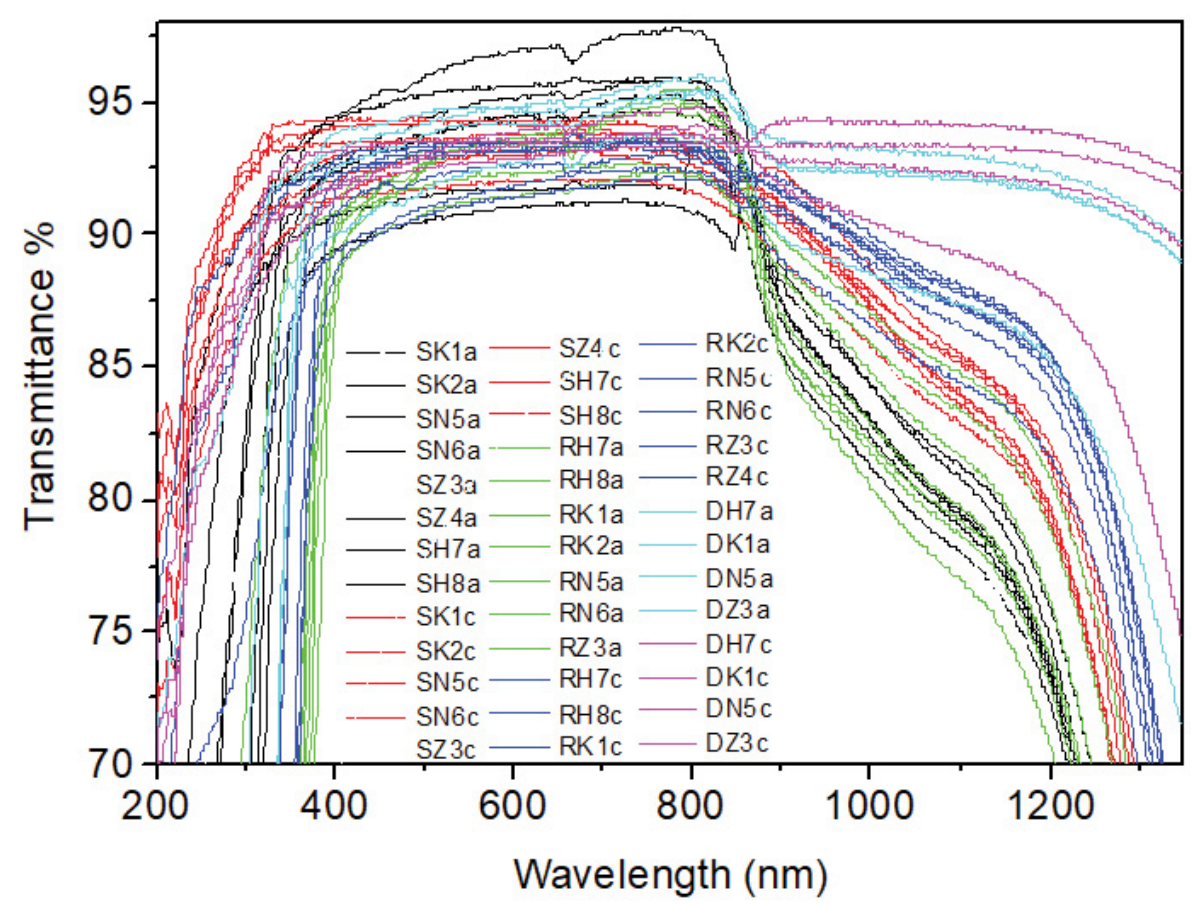

Figure 3: The transparent spectra of slow and rapid KDP and DKDP.

Table 1: Laser induced damage of KDP and DKDP crystals.

\begin{tabular}{|l|l|l|l|}
\hline Samples & Energy(mJ) & Energy density $\left(\mathbf{J} / \mathbf{c m}^{2}\right)$ & Power density $\left(\mathbf{G W} / \mathbf{c m}^{2}\right)$ \\
\hline DN5 & $192 \sim 240$ & $24.4 \sim 30.6$ & $2.44 \sim 3.06$ \\
\hline DH7 & 240 & 30.6 & 3.06 \\
\hline RH7 & $95 \sim 143$ & $12.1 \sim 18.2$ & $1.21 \sim 1.82$ \\
\hline RK2 & 143 & 18.2 & 1.82 \\
\hline SK2 & 220 & 28 & 2.8 \\
\hline SK1 & $143 \sim 200$ & $18.2 \sim 25.5$ & $1.82 \sim 2.55$ \\
\hline
\end{tabular}

rections) $>$ DKDP (along $c$ directions) $>$ traditional growth KDP (along $a$ directions) $>$ DKDP (along $a$ directions) $>$ Rapid growth KDP (along $c$ directions) > Rapid growth KDP (along $a$ directions). For region II, the average transmittance of all samples is higher than $90 \%$, and they were ranked in order of DKDP (along $a$ directions) > DKDP (along $c$ directions) $>$ traditional growth KDP = Rapid growth KDP. We note that an inflection point appeared at about $850 \mathrm{~nm}$ for all samples, except for DKDP measuring along $c$ direction. Regarding III, the average transmittance of all samples except for DKDP is lower than $90 \%$, and they were ranked in order of DKDP (along $c$ directions) $>$ DKDP (along $a$ directions) $>$ Rapid growth KDP (along $c$ directions) $>$ traditional growth KDP (along $c$ directions) $>$ Rapid growth $\mathrm{KDP}$ (along $a$ directions) $=$ traditional growth KDP (along $a$ directions). For region $\mathrm{V}$, the transmittances of KDP decrease sharply, while for DKDP de- crease slowly to $80 \%$ at about $1500 \mathrm{~nm}$ and then fell sharply too. The results indicated that the average transmittance of traditional growth KDP is higher as we expected than those of rapid growth KDP, which however seems to be inconsistent with the measured extinction ratios. In other words, the higher the transmittance is, the lower the measured extinction ratio. It was suggested that the optical properties could be well characterized by transmittance than extinction ratio since the extinction ratio is more sensitive to the micro defects, sample orientations or other factors, whereas the transmittance reflects macro and statistical effect.

\section{Laser damage threshold}

The beam diameter was minimized by a lens system to $1 \mathrm{~mm}$. The measured results were listed in Table 1. From this table it can be seen that the laser damage threshold of DKDP is highest up to $240 \mathrm{~mJ}$. 

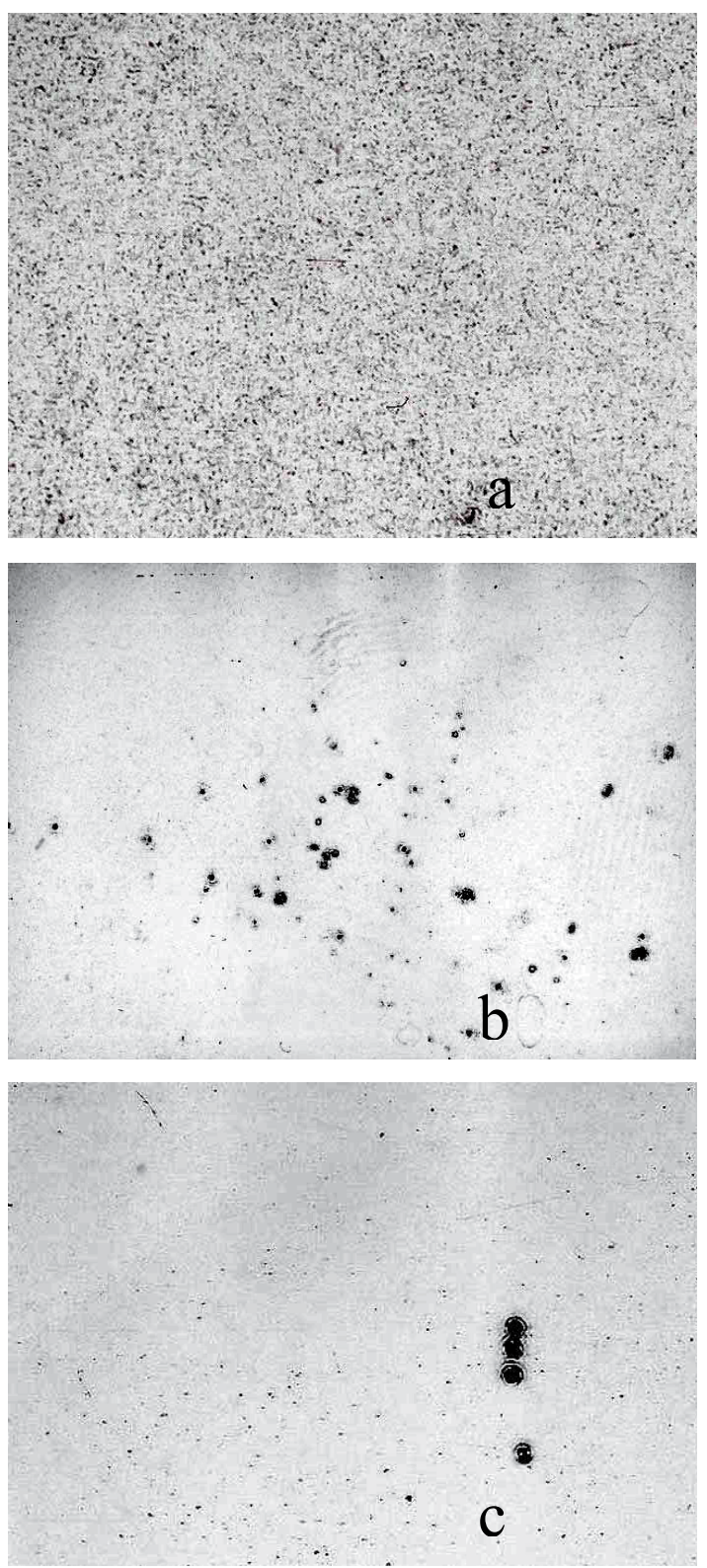

Figure 4: Scattering particles observed in typical: (a) Traditional; (b) Rapid growth KDP and; (c) DKDP crystals.
The values of rapid growth KDP are obvious lower than those of DKDP and traditional growth KDP and the highest is only up to $143 \mathrm{~mJ}$. Combined with above measured other optical or electrical parameters, it was found that the laser damage threshold is approximately proportional to the extinction ratio, optical transmittance, and whereas inversely proportional to the conductivity.

\section{Distribution of optical scattering particles}

Figure $4 a$, Figure $4 b$ and Figure $4 c$ shows the photographs of scattering particles observed in typical traditional, rapid growth KDP and DKDP crystals. From Figure $4 a$ it could be learned that the density of optical scattering particles in traditional growth KDP is very high although the sizes are small, which may be responsible for lowing of the above measured extinction ratios. The density of scattering particles in rapid growth KDP as shown in Figure $4 \mathrm{~b}$ is low and the distribution is somewhat localized although the sizes are relatively large, which leading to the accidental influence on the extinction ratio measuring. From Figure $4 \mathrm{c}$ it was noted that the density of scattering particles in DKDP is very low although the sizes are $2 \sim 5$ times larger than that of rapid growth KDP crystals, indicating that the right extinction ratios could be obtained if the incident beam avoids the scattering particles.

In addition, all the samples were thermal annealed in air, vacuum, $\mathrm{N}_{2}$ and $\mathrm{H}_{2}$, and then the extinction ratio, conductivity, absorption spectrum, laser damage threshold as well as optical scattering particles were measured and compared before and after annealing. It was found that the optical quality can be improved by thermal annealing in vacuum and $\mathrm{H}_{2}$, and whereas no obvious effect in air and
(A)

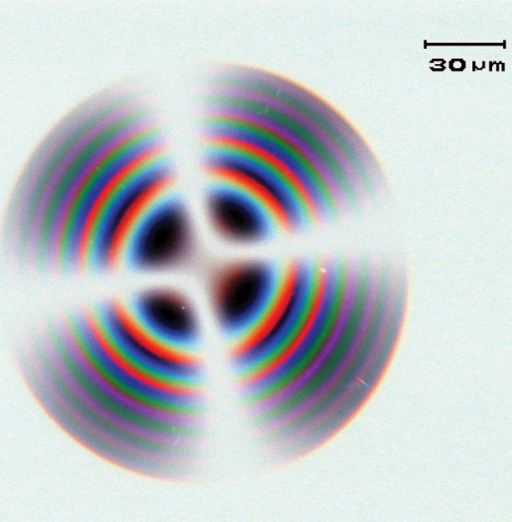

(b)

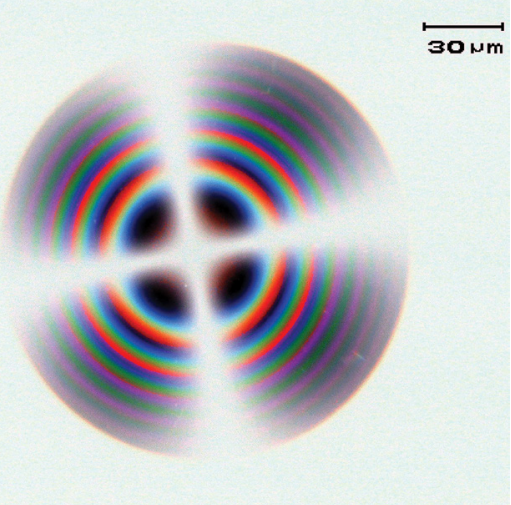

Figure 5: Taper optical interference at the edge of KDP crystals before and after $\mathrm{H}_{2}$ annealing. 
$\mathrm{N}_{2}$. As an example, the taper optical interference patterns observed in the edge of typical traditional growth KDP before and after thermal annealing in $\mathrm{H}_{2}$ were shown in Figure 5. It is obvious that the internal stress in crystals decreased by $\mathrm{H}_{2}$ annealing and the optical quality is therefore improved. The similar results for other optical and electrical parameters have also been obtained, and in this paper the detailed descriptions were omitted.

\section{Conclusions}

The optical and electrical measurements, including the extinction ratio, conductivity, absorption spectrum, laser damage threshold as well as optical scattering particles, were performed on the traditional, rapid growth KDP and DKDP crystals, respectively. The thermal annealing in air, vacuum, $\mathrm{N}_{2}$ and $\mathrm{H}_{2}$ were also performed on all samples. The effect on optical properties of growth conditions and the correlations among various optical parameters were investigated. It was concluded that the laser damage threshold is approximately proportional to the extinction ratio, optical transmittance, and whereas inversely proportional to the conductivity, and we firstly found that the optical quality can be improved by thermal annealing in vacuum and $\mathrm{H}_{2^{\prime}}$ and whereas no obvious effect in air and $\mathrm{N}_{2}$.

\section{References}

1. C Mao, G Zhou, X Sun, G Li, J Wang, et al. (2020) Optical and thermal properties of I-tartaric doped KDP crystals. Optik 203: 163967.

2. D Wang, G Liu, C Shen, C Chen, Y Wang, et al. (2020)
Experiment and numerical simulation of the influence mechanism of kinetic factors on rapid growth of KDP crystal. Ceramics International 46: 538-544.

3. J West (1930) A quantitative X-ray analysis of the structure of potassium dihydrogen phosphate $(\mathrm{KH}-$ 2PO4). Zeitschrift für Kristallographie 74: 306.

4. G Li, G Zheng, Y Qi, P Yin, E Tang, et al. (2014) Rapid growth of a large-scale (600 mm aperture) KDP crystal and its optical quality. High Power Laser Science and Engineering 2: e2.

5. Z Xinxin, Y Liwang, Z Guozong, S Genbo, $\mathrm{H}$ Youpin, et al. (2011) The rapid growth of large-scale KDP single crystal in brief procedure. Journal of Crystal Growth 318: 700-702.

6. WK Peng, Y Shi (2011) S substituting for $P$ point defect-induced laser damage in KDP crystals. Acta Physica Sinica 60: 097401.

7. KP Wang, CS Fang, JX Zhang, CS Liu, RI Boughton, et al. (2005) Interstitial oxygen induced laser damage in KDP. Phys Rev B 72: 184105.

8. G Pei, L Ye, W Du, H Yan, X Xu (2020) Optimization of second-harmonic generation in large-aperture KDP crystals using multipoint support mounting configurations. Optical Engineering 59: 016104.

9. YB Zheng, XD Zhou, RS Ba, J Li, H Lei, et al. (2020) Microscopic characterization of bulk damage resistance of DKDP nonlinear crystals. Optics \& Laser Technology 121: 105672.

10.J Zhang, Z Tian, K Wang (2020) Optimal design of the annealing furnace and quality improvement for KDP crystals. Optik 217: 164874. 